\title{
Brief Reports
}

Quantifying the Contribution of VA Service-Connected

Disability Pensions to the "Check Effect"

Daniel J. Luchins, MD

John F. McCarthy, PhD, MPH

Jennifer J. Mach

Neil Jordan, PhD

Dr. Luchins is affiliated with the Department of Psychiatry, Jesse Brown Department of Veterans Affairs (VA) Medical Center, 820 S. Damen Ave, A116, Chicago, IL 60612 (email: daniel.luchins@va.gov). He is also with the Department of Psychiatry, University of Illinois at Chicago. Dr. McCarthy and Ms. Mach are with the VA National

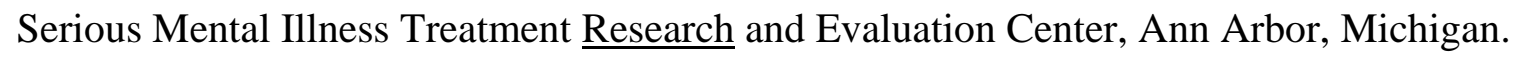
Dr. Jordan is with the Department of Psychiatry and Behavioral Sciences, Feinberg School of Medicine, Northwestern University, Chicago. He is also with the Center for Management of Complex Chronic Care, Hines VA Hospital, Hines, IL.

Objective: The study attempted to quantify the effect of receiving a disability pension check at the beginning of the month on the timing of substance-related hospitalizations in Department of Veterans Affairs (VA) facilities. Methods: All substance-related hospitalizations during fiscal year 2005 were identified in the VA National Psychosis Registry (13,402 hospitalizations among 8,813 individuals) and in a random sample of VA patients without serious mental illness (689 hospitalizations among 508 individuals). Multivariable logistic regression examined the impact of pension check receipt on timing of hospitalizations while controlling for other predictors of substance-related hospitalizations. Results: Receipt of a service-connected pension was associated with an increased risk of admission during the first two weeks of the month (odds ratio=1.08; 95\% confidence interval=1.01-1.16). Conclusions: Staggering the disbursement of VA disability pensions (sending half the checks at the beginning and half in the middle of the 
month) might modestly reduce peak demand for substance-related inpatient services. (Psychiatric Services 62:XXXXXX, 2011)

In a study of 105 veterans with schizophrenia and cocaine abuse at a Department of Veterans Affairs (VA) medical center, Shaner and colleagues (1) demonstrated a cycle of cocaine use and psychiatric hospitalization that coincided with receipt of disability benefits checks at the beginning of each month. They observed monthly peaks and troughs, with almost two-thirds of admissions occurring in the first half of the month. These findings were interpreted as establishing a causal relationship between receipt of public support payments, substance abuse, and utilization of psychiatric services that has been referred to as the "check effect" and may have influenced the federal government's decision to eliminate disability benefits for substance use disorders in 1997.

However, subsequent research suggests that these payments affect recipients' timing of use but not necessarily their overall use of substances or psychiatric services. The Social Security Administration's elimination of disability benefits for individuals with substance use disorders did not affect the number of visits to psychiatric emergency departments by persons in this group (2). Moreover, studies of homeless veterans with serious mental illness and co-occurring substance-related disorders have shown no difference in levels of substance use among those who receive public support payments and those who do not (3) and no increase in substance use after initiation of benefits (4). On the other hand, several studies have confirmed a pattern of increased substance abuse and psychiatric service utilization during the earlier part of the month $(5,6)$.

If this temporal pattern of substance abuse and use of psychiatric services is partly attributable to receipt of disability checks, then such patterns might be mitigated by altering the timing of check disbursement. The VA currently disburses all serviceconnected disability pension checks at the beginning of each month. However, disbursement of checks to half the recipients at the beginning of the month and to the other half on the 15th might smooth out the peaks and troughs in service use, thereby reducing the need for fixed resources, such as professional staff or psychiatric beds. 
Previous VA studies that showed a large check effect $(1,7)$ did not attempt to quantify the extent to which the effect was attributable to receipt of a VA pension check or to other financial events that coincide with the beginning of the month, such as receipt of Supplemental Security Income (SSI), Social Security Disability Insurance (SSDI), employment checks and rent, utility, and child support payments. These other events might also affect substance abuse and use of psychiatric services (5) but would be unaffected by a change in VA policy. Further, previous studies were limited to small catchment areas and did not assess whether the check effect was concentrated among veterans with serious mental illnesses or those with multiple, recent hospitalizations. The study reported here used national VA health system data to examine these issues.

\section{Methods}

Our study examined VA data from three sources: the VA National Psychosis Registry, which contains all inpatient and outpatient encounters for all VA patients with diagnoses of serious mental illness, defined as schizophrenia, bipolar disorder, or other psychotic disorders; inpatient and outpatient data for a national random sample of VA patients with service utilization in fiscal year (FY) 2005 (8); and VA administrative data regarding service-connected disability and pension status. The Ann Arbor VA Medical Center Institutional Review Board approved data management and analyses as part of a larger project examining resource utilization among VA patients with mental illness.

We identified all substance-related hospitalizations during the first four weeks of each month in FY 2005 for two cohorts: all VA patients with a serious mental illness diagnosis (13,402 admissions among 8,813 individuals) and a random sample of VA patients who used a VA facility in FY 2005, excluding those with a serious mental illness (689 admissions among 508 individuals).

Using multivariable logistic regression with generalized estimating equations (9) to account for clustering by patient, we examined the timing of hospitalizations for substance abuse or dependence. Admissions were categorized as having occurred in the first 14 days of the month or in the second 14 days of the month. Our analyses excluded 
hospitalizations that occurred after the $28^{\text {th }}$ day of the month. Independent variables included patient age, gender, race-ethnicity (coded as white or nonwhite), marital status, homelessness at some point in FY 2005, service-connected disability status of at least $10 \%$ (the minimum for receipt of a service-related pension), and whether the substancerelated hospitalization was preceded by an inpatient admission in the previous six months.

\section{Results}

Among the 9,321 veterans in the total sample, there were 14,091 substance-related hospitalizations in FY 2005 (mean=1.51 1 1.05). A total of 4,676 hospitalizations (33\%) involved individuals with service-connected disability pensions. The mean age for the total sample was $49 \pm 8$ years. A total of 13,451 (96\%) of the admissions were of men, 8,376 (59\%) were of white veterans, 1,879 (13\%) were of currently married veterans, 8,818 (63\%) were of homeless veterans, and in the case of 8,221 (58\%) admissions the individual had had a hospitalization in the previous six months.

Of the 4,676 admissions of veterans with service-connected disability pensions, 2,532 (54\%) occurred in the first two weeks of each month and 2,144 (46\%) in the next two weeks. Of the $\underline{9,415}$ admissions of veterans without service-connected pensions, 4,921 (52\%) occurred in the first two weeks and 4,494 (48\%) in the next two weeks. Although both groups demonstrated a check effect - that is, more admissions in the beginning of the month—multivariable logistic regression indicated that having a service-connected pension was associated with significantly greater odds that an admission occurred during the first two-week period (odds ratio $[\mathrm{OR}]=1.08$, 95\% confidence interval [CI] $=1.01$ 1.16). This pattern was also more likely among veterans who were homeless $(\mathrm{OR}=1.08$, $95 \% \mathrm{CI}=1.01-1.17$ ) or nonwhite (OR=1.09, 95\% CI=1.01-1.16). Having a diagnosis of a serious mental illness or having a hospitalization in the prior six months was not significantly associated with the timing of substance-related hospitalizations.

\section{Discussion}


This analysis of large national samples of veterans hospitalized for substance-related disorders indicated an overall increase in the number of admissions during the first two weeks of the month, consistent with the check effect hypothesis. However, this pattern did not differ between those with or without a serious mental illness or between those who did or did not have a recent prior hospitalization. These findings suggest that the check effect is not restricted to the most seriously ill or those with multiple, recent hospitalizations. However, as hypothesized, admissions were more likely to occur in the first two weeks of the month among individuals who were receiving a service-connected disability pension.

This is the first study to quantify the degree to which receiving a particular type of income might contribute to the check effect. An implication of this finding is that instituting a VA-wide policy to change the timing of disability check disbursement (such as sending out half the checks at the beginning of the month and half in the middle of the month) might modestly reduce peak demand for inpatient hospital beds, which may allow a reduction in the level of ongoing fixed resources necessary to serve this population. The VA currently issues checks several times per month for a range of other purposes (for example, VA employees receive paychecks twice monthly); therefore, implementation of such a policy may not involve substantial barriers.

Another practical feature of such a policy is that it would not depend on the action of clinicians, unlike the establishment of a representative payeeship. VA clinicians are extremely reluctant to recommend establishing a payeeship, even for substance-abusing patients with known money management problems who might benefit from the assignment of a payee (10). Also, because a policy that changed the disability check disbursement schedule would be applied to all veterans receiving service-connected disability pensions, it would not be subject to the criticism that it is discriminatory to patients with mental illnesses or substance use disorders.

Our analysis also identified two additional variables_-homelessness and being nonwhite - that were associated with an $8 \%$ and $9 \%$ greater likelihood, respectively, of 
substance-related admissions during the first two weeks of the month. This may reflect the influence of other income received at monthly intervals. Further research is needed to test this hypothesis.

This study focused on the component of the check effect that may be ascribable to receipt of a VA service-connected pension. It did not examine the impact of monthly support payments (SSDI or SSI) disbursed by other federal agencies. We expect that these payments may also contribute to the overall check effect. If staggering the disbursement of VA service-connected disability pensions reduces monthly peaks in demand for mental health services, a federal governmentwide policy to stagger the disbursement of support payments might allow for additional reductions in fixed resources and periodic overcrowding in medical centers.

\section{Conclusions}

The study's findings indicate that veterans who received a VA service-connected disability pension were more likely to have a hospital admission for a substance-related disorder during the first two weeks of the month than in the next two weeks. This raises the possibility that staggering the timing of check disbursement may mitigate the check effect and save resources and reduce monthly periods of overcrowding.

\section{Acknowledgments and disclosures}

The views expressed in this article are those of the authors and do not necessarily reflect the position or policy of the Department of Veterans Affairs or the United States government.

$\underline{\text { The authors report no competing interests. }}$

\section{References}

1. Shaner A, Eckman TA, Roberts LF: Disability income: cocaine use and repeated hospitalization among schizophrenic cocaine abusers. New England Journal of Medicine 333:777-783, 1995 
2. Catalano R, McConnell W: Psychiatric emergencies: the check effect revisited. Journal of Health and Social Behavior 40:79-86, 1999

3. Frisman LK, Rosenheck R: The relationship of public support payments to substance abuse among homeless veterans with mental illness. Psychiatric Services 48:792-795, 1997

4. Rosen MI, McMahon TJ, Lin HQ, Rosenheck RA: Effect of Social Security payments on substance abuse in a homeless mentally ill cohort. Health Services Research 41:173-191, 2006

5. Catalano R, McConnell W, Forster P, et al: Does the disbursement of income increase psychiatric emergencies involving drugs and alcohol? Health Services Research 35:813-823, 2000

6. Halpern SD, Mechem C: Declining rate of substance abuse throughout the month. American Journal of Medicine 110:347-351, 2001

7. Grossman LS, Willer JK, Miller NS, et al: Temporal patterns of veterans' psychiatric service utilization, disability payment, and cocaine use. Journal of Psychoactive Drugs 29:285-290, 1997

8. McCarthy JF, Valenstein M, Blow FC: Residential mobility among patients in the VA health system: association with psychiatric morbidity, geographic accessibility, and continuity of care. Administration and Policy in Mental Health 34:448-455, 2007

9. Cui J: QIC program and model selection in GEE analysis. Stata Journal 7:209220, 2007

10. Rosen MI, Rosenheck RA, Shaner A, et al: Veterans who may need a payee to prevent misuse of funds for drugs. Psychiatric Services 53:995-1000, 2002 